\title{
Ion Charge State Fluctuations in Vacuum Arcs
}

\author{
André Anders ${ }^{1}$, Kentaro Fukuda ${ }^{2}$, and George Yu. Yushkov ${ }^{3}$ \\ ${ }^{1}$ Lawrence Berkeley National Laboratory, University of California, Berkeley, California 94720, USA \\ ${ }^{2}$ Nippon Sheet Glass Co. Ltd., Tsukuba, Ibaraki, 300-2635, Japan \\ ${ }^{3}$ High Current Electronics Institute, Russian Academy of Sciences, Tomsk, 634055, Russia
}

\begin{abstract}
Ion charge state distributions of cathodic vacuum arcs have been investigated using a modified time-of-flight method. Experiments have been done in double gate and burst gate mode, allowing us to study both systematic and stochastic changes of ion charge state distributions with a time resolution down to $100 \mathrm{~ns}$. In the double gate method, two ion charge spectra are recorded with a well-defined time between measurements. The elements $\mathrm{Mg}, \mathrm{Bi}$, and $\mathrm{Cu}$ were selected for tests, representing metals of very different properties. For all elements it was found that large stochastic changes occur even at the limit of resolution. This is in agreement with fast changing arc properties observed elsewhere. Correlation of results for short times between measurements was found but it is argued that this is due to velocity mixing rather than due to cathode processes. The burst mode of time-of-flight measurements revealed the systematic time evolution of ion charge states within a single arc discharge, as opposed to previous measurements that relied on data averaged over many pulses. The technique shows the decay of the mean ion charge state as well as the level of material-dependent fluctuations.
\end{abstract}




\section{Introduction}

For many years it is well known that cathodic vacuum arc plasmas contain multiple ion charge states [1-4]. The charge state distributions are material dependent and the ion mean charge can be reduced by the presence of background gas [5-8] or enhanced by magnetic fields [9-11]. The influence of the magnetic field is significant for external fields while the magnetic self-field can be neglected as long as the current is relatively low ( $<200 \mathrm{~A}[9]$ and $<500 \mathrm{~A}[10])$. The self-field must be taken into account for highcurrent $(\mathrm{kA}) \operatorname{arcs}[10]$.

A common way to obtain time-resolved data is to measure at different times after arc triggering using for example time-of-flight (TOF) charge-to-mass spectroscopy $[3,12]$. The TOF technique has a time resolution that is limited by the duration of the gating pulse, which is typically $100 \mathrm{~ns}$. It was found that the charge states are higher at the beginning of each arc, decaying to steady-state values after several $100 \mu \mathrm{s}$ [11] or even milliseconds [13].

In order to arrive at the above conclusions, many individual measurements were made and averaged. For example, one can find data on average ion charge states $[2,3]$, average burning voltages [14, 15], average velocities [11], or average velocity distribution functions [16]. The arc plasma is known to be "noisy," that is, all parameters fluctuate with large amplitude (some up to $100 \%$ of the average value), which can be associated with the explosive nature of cathode spot ignition and plasma production [17-22]. Additional sources of "noise" may exist, such as current-driven plasma instabilities [23, 24].

While research until now focused on finding trends and models for averaged distributions and other arc data, relatively little attention was paid to fluctuations or "noise," although the fluctuating signals are likely to contain information on plasma formation processes. Our current research tries to reduce this shortcoming, and this report focuses on fluctuations of ion charge states.

The noise in ion charge state is not only of fundamental interest. Cathodic arc ions are used for film formation in energetic condensation of cathodic arc plasma. They are also used as feedstock material in vacuum arc ion sources, also known as metal vapor vacuum arc or "Mevva" ion sources, which are used, for example, to inject ions into accelerators [13]. In the field of accelerators, fluctuations are of great concern and efforts have been made to reduce them as much as possible [25, 26]. 
In the field of cathodic arc coatings, noise is usually ignored with the argument that the duration of deposition is much longer than any characteristic time of fluctuations, hence averaging appears to be justified. However, when looking at atomistic mechanisms of film growth, fluctuations may have critical importance by providing multiply charged, high-energy ions, which are critical for densification and ion damage to crystallites but appear negligible when looking at time-averaged distributions.

When measurements are repeated under macroscopically identical conditions, different results are obtained. Therefore, in order to say anything, researchers opted to average many measurements and derive conclusions on average data, especially when discussing plasma parameters like ion charge state distributions $[10,27-30]$. The time between individual measurements was usually large, often 100s of milliseconds or more. In many cases [10, 28-30], pulsed arcs were used, and one TOF spectrum was acquired per arc pulse. To our knowledge, no information is available on how fast ion charge states fluctuate. Therefore, we aim here to dramatically reduce the time between TOF measurements and to look for dynamic changes and stochastic properties of the signal.

\section{Experimental}

The experiments were made at the vacuum arc ion source "Mevva V" at Lawrence Berkeley Lab; the source has been described elsewhere [3]. Figure 1 schematically shows the geometric and electrical setup. A cathode rod of $6.25 \mathrm{~mm}$ diameter is placed in a ceramic tube allowing a cathode spot to burn only on the rod's front face. The plasma produced at cathode spots expanded through an annular anode and arrived after $r=138 \mathrm{~mm}$ drift at a three-grid ion extraction system, which is a conventional, multi-aperture acceleration-deceleration system [31]. The extraction system is needed to form an ion beam that can be used for the TOF diagnostic of ion charge states. The mass of ions is known by the given cathode material, and hence charge states can be immediately interpreted from the TOF ion spectrum. The arc current was pulsed; each pulse had a duration of $250 \mu$ s (a property of the 8 -stage, $1-\Omega$ pulse-forming network, PFN), and the amplitude could be adjusted between 50 A and 500 A by changing the PFN's charging voltage. The vacuum base pressure was about $8 \times 10^{-5} \mathrm{~Pa}$; no working gas was used.

In contrast to previous work $[10,12,28-30]$, the TOF instrument is now equipped with a gate pulse generator that can deliver two or more gate pulses of max. $3.5 \mathrm{kV}$, with arbitrarily small delay to the 
previous pulse. To obtain a clear ion signal, however, the gate pulse duration should not be less than 100 ns, and hence a practical time resolution of about $150 \mathrm{~ns}$ is obtained. This limitation did not appear to be detrimental because ion velocity mixing represented a much larger factor in the investigation of cathode processes, as explained below.

Ions extracted from the plasma form a beam; the ion energy is given by $E_{i}(Q)=Q e V_{\text {extr }}$, where $Q$ is the charge state number, $e$ is the elementary charge, and $V_{\text {extr }}$ is the extraction voltage, which was typically $40 \mathrm{kV}$ in the present experiments. The very small contribution of plasma streaming velocity to the kinetic energy of extracted ions can be neglected. Once extracted, ions of higher charge state have greater kinetic energy, and knowing that they have the same mass, higher charge state translate into higher velocity according to

$$
v_{i}(Q)=\sqrt{\frac{2 Q e V_{e x t r}}{m_{i}}} .
$$

Therefore, ions of higher charge state arrive earlier at the detector, which is the basis for TOF measurements. The detector was a Faraday cup with magnetic suppression of secondary electrons. TOF gate pulse and Faraday cup current were recorded by a digital storage oscilloscope with $500 \mathrm{MHz}$ bandwidth and up to $1 \mathrm{Gs} / \mathrm{s}$ sample rate. The width of each record was 50,000 data points.

In a first round of experiments, a sequence of two gate pulses was used. The time between the two gate pulses could be freely adjusted. Figure 2 shows a typical arc discharge pulse and an example of timing the two probing pulses with respect to the arc pulse and with respect to each other. An example of two TOF spectra for copper is shown in Figure 3. One can clearly see that the spectrum contains charges 1 to 4 , with subtle differences between the spectra.

Data generation and acquisition was made as follows. The first gate pulse was set at $t_{\text {gatel }}=150 \mu \mathrm{s}$ after arc triggering. This choice was made to avoid the well-known large shifts in the charge state distribution that occur in the first $150 \mu$ s after triggering [32,33]. Ideally, one would look at even later times because it takes milliseconds to reach quasi-steady-state distributions [13], however, the arc pulse of the "Mevva V" system was only $250 \mu$ s long. A choice of $150 \mu$ s for the first measurement allowed us to use a delay $\Delta t=t_{\text {gate } 2}-t_{\text {gate }}$ of up to $100 \mu$ s for the second gate pulse. This delay was kept constant when collecting a set of data consisting of 25 pairs of charge spectra recorded under macroscopically identical 
conditions. Then, the time difference $\Delta t$ was varied in order to study the time-dependent correlation between subsequent spectra, should such correlation exist.

Each spectrum shows peaks caused by the electrical current of the specific charge states. Since the width is the same for all signals, it is sufficient to consider their amplitudes $A_{P Q}$, where the indices $P$ and $Q$ stand for the gate pulse number and charge state number, respectively. The corresponding particle density, expressed in relative units, can be written as

$$
N_{P Q}=A_{P Q} / Q
$$

The difference between spectra obtained by gate pulses 1 and 2 can be expressed by the absolute scattering of the ion density with charge $Q$,

$$
\Delta N_{Q}=N_{2 Q}-N_{1 Q}
$$

In another round of experiments, a burst of many gate pulses was generated such as to obtain a burst of many charge spectra showing the evolution of charge state distribution in a single arc discharge. While the first approach with double pulses purely focused on stochastic properties and statistically relevant correlation, the burst method would also show the evolution of charge states in time, with random stochastic noise superimposed on dynamic changes.

\section{Results}

\subsection{Double pulse experiments}

The elements $\mathrm{Mg}$ and $\mathrm{Bi}$ were initially selected for these measurements because their ion spectra contain only two peaks, corresponding to charge $1+$ and $2+$, therefore measurements and interpretation are particularly simple and transparent. The measurements were later complemented with $\mathrm{Cu}$ whose spectrum contains charge states $1+$ to $4+$, as shown in Figure 3.

Figures 4-6 show the results for $\mathrm{Mg}, \mathrm{Bi}$, and $\mathrm{Cu}$. One can see that changes in the charge states occur all the way to the highest time resolution for all three materials investigated, although the magnitudes of changes were smaller when only a short time elapsed between measurements. The data for copper (figure 6) appear to have a "hole" in the range around $0.5 \mu \mathrm{s}$. Information in this time region was not acquired because the peaks of higher charge of the second spectrum overlapped with the peaks of lower charge state from the first spectrum, which made quantification difficult. 
In figures 4-6, absolute changes of the signal amplitude are displayed. Absolute fluctuations are important to accelerator injectors. For other applications, fluctuations of the charge state distribution may be of greater importance than absolute fluctuations. If the charge state amplitudes changed proportionally for all charge states, figures 4-6 would show large $\Delta N_{Q}$ although the charge state ratios remained the same. Therefore, in another way of presenting results, one should look at changes of the mean ion charge state when measuring with two immediately successive gate pulses. We define the mean charge state for a single measurement with number $i$ according to

$$
\bar{Q}_{i}=\sum_{Q=1}^{Q_{\max }} A_{i Q} / \sum_{Q=1}^{Q_{\max }} N_{i Q}
$$

where $Q_{\max }$ is the maximum charge state present in the plasma, and $i=1,2,25$ in our experiments. For each set of measurements, consisting of pairs of 25 spectra, one can determine a set of changes representing the 25 differences of the mean charge states:

$$
\Delta \bar{Q}_{i}(\Delta t)=\bar{Q}_{i}\left(t_{\text {gate }}\right)-\bar{Q}_{i}\left(t_{\text {gatel }}\right) .
$$

One may further define the difference in the mean ion charge state averaged over 25 measurements made at macroscopically identical conditions,

$$
\langle\Delta \bar{Q}(\Delta t)\rangle=\sum_{i=1}^{i_{\max }} \Delta \bar{Q}_{i}(\Delta t) / i_{\max }
$$

where $i_{\max }=25$. To clarify the situation, a bar represents averaging over charge states while a bracket indicates averaging over a number of repeated measurements.

Further, one may determine the standard deviation for this set of differences, which is by definition

$$
\sigma(\Delta t)=\sqrt{\frac{1}{i_{\max }-1} \sum_{i=1}^{i_{\max }}\left(\Delta \bar{Q}_{i}(\Delta t)-\langle\Delta \bar{Q}(\Delta t)\rangle\right)^{2}} .
$$

Figure 7 shows an evaluation of the same data presented in figures 4-6. Figure 7 indicates that there exist two time domains, one where measurements are close (small standard deviation) and one where charge states changed a lot. The transition between these regimes appears to depend on the material and especially clear for the heavy material bismuth. We suspect an effect associated with the finite plasma flow time prior to ion extraction, and therefore the experiment was repeated for bismuth with the plasma expansion zone 
shortened from the original $138 \mathrm{~mm}$ to $69 \mathrm{~mm}$. The result is shown in figure 8 , which seems to confirm our hypothesis.

Part of the noise is thought to be generated by the many spot ignition events. Because it is known that higher arc current causes more emission sites to exits, one may expect that more "noise generators" create a decrease in the relative level of fluctuations. Therefore, measurements have been made with magnesium at various arc currents, which indeed showed a tendency of lower noise at higher current (Fig. 9).

\subsection{Burst pulse experiments}

For the multiple gate experiment, a burst of gate pulses was generated that covered the entire arc pulse, as shown in figure 10. Each gate pulse generated a complete charge state spectrum, as shown in figures 11 and 12 with greater time resolution. This kind of experiment allowed us to look for systematic and stochastic changes in the charge state distribution.

Figure 13 shows examples of the evolution of ion charge state distributions for $\mathrm{Mg}, \mathrm{Bi}$, and $\mathrm{Cu}$. In contrast to previous work, the mean ion charge state is obtained here by averaging the charge state distribution at a given time and not, as in previous work, over many measurements. Each measurement gave a curve like the examples in Figure 13, characterized by a material-dependent decay and level of stochastic noise.

\section{Discussion}

The measurements presented here confirm a number of well-known cathodic arc properties as well as describe new observations. Considering the data shown in figures 4-6, one can see that repeated measurements always show variation, even for the shortest time differences measured (150 ns). This is an indication that fundamental processes of plasma production at cathode spots have features whose characteristic time is shorter than $150 \mathrm{~ns}$. This is not surprising because fast changes and fluctuations have

previously been observed in other arc parameters, such as crater formation [34,35], voltage and light emission [20] and absorption [36]. 
Another feature seen in figures 4-6 is that, on the average, more fluctuation data points are below the $\Delta N_{Q}=0$ line than above. This is just another indication that ion charge states have a systematic shift to lower values until steady-state (in the time-averaged sense) is reached. The shifts may have various origins, which are still subject to research, including change of the surface state of the cathode due to erosion by the cathode spot, heating of the cathode surface, plasma-cathode interaction, and initially greater specific energy input in the plasma. Recently, a new interpretation was put forward taking into account the relatively slow evolution of neutrals in the discharge volume, and charge exchange collisions between neutrals and ions [33].

As indicated in figures 7 and 8 , changes in charge state distributions were small when measurements were made with little time in between. This effect was particularly pronounced for the heavier cathode materials. At this point one could suspect that the observed time is characteristic for the material, perhaps one of the long-sought characteristic times for plasma production at cathode spot. However, one should realize that the experimental setup involves a natural time characteristic on its own, namely the time-of-flight of ions from the location of their "birth," the cathode spot, to the ion extractor system. If all ions emitted from the cathode spot had exactly the same velocity, the interpretation of a characteristic cathode phenomenon would have merit because information from the cathode spot would be preserved by the ion flow and "handed over" from the plasma to the ion beam. That, however, is not the case. Ions emitted from the cathode spot region have a velocity distribution, $f_{i}(v)$, such as the one shown in figure 14 for $\mathrm{Mg}$. Due to the non-zero width of the velocity distribution, the information on cathode processes is smeared out and mixed, and thus the extracted ion current becomes correlated over a time determined by the width of the velocity distribution function. The reason is that faster ions overtake slower ions generated in earlier emission events, i.e., ions arriving at the extractor grid are "velocity phase mixed." As a result, whenever we investigate the charge state distribution sufficiently fast such that velocity phase mixing matters, we are looking at the ion emission events with a method that inherently features correlation. The ion density at the extractor can be described by a correlation function of the type

$$
n_{i}(r, t)=\int_{0}^{t} g_{i}(r, t-\tau) S(\tau) d \tau
$$


where $r=138 \mathrm{~mm}$ is the distance of the extractor form the plasma source (cathode surface); $S(\tau)$ is the time-dependent source intensity (in $\mathrm{s}^{-1}$ ), and

$$
g_{i}(r, t) \approx\left(\frac{m_{i}}{2 \pi k T}\right)^{3 / 2} \frac{1}{t^{3}} \exp \left(-\frac{m_{i}}{2 k T} \frac{r^{2}}{t^{2}}\right)
$$

is a density function which was derived assuming collisionless expansion from a point source of Maxwellian particles [20,37]. The plasma production can be thought of a sequence of instantaneous pulses,

$$
S(t) \approx S \delta(t)
$$

where $\delta(t)$ is the Dirac function. The important physical point is that ions available for extraction at time $t$ depend on the plasma generation over a range of times prior to $t$. Future research should bring further clarification by specifying the relation between the experimental ion velocity distribution function, $f_{i}(v)$, as shown in figure 14, and the density function, $g_{i}(t)$, derived for simplified assumptions.

In figure 9, it was shown that the level of noise was reduced when the arc current was increased. This does not come at a surprise since it is assumed that fast cathode processes are responsible for causing "noise" and that the number of plasma-generating emission sites increases with current. From the wellknown theory of noise one should expect that that noise level decreases with the square root of the number of noise generators, hence the standard deviation of the data should scale with reciprocal square root of the arc current:

$$
\sigma \square 1 / \sqrt{n}=1 / \sqrt{I_{\text {arc }} / I_{E S}} .
$$

The typical current per emission site, $I_{E S}$, is about $0.8 \mathrm{~A}$ for magnesium [38]. This dependence is plotted in figure 9, with good agreement to experimental data.

Finally, another critical point of the measurement method should be pointed out. This concerns the possible distortion of information by ion optics effects. If we are interested to use the TOF method with ion extraction to diagnose the plasma, we assume that the detected ion beam composition is an image of the actual ion concentration in the plasma. As with all imaging system, there is only a limited level of fidelity. Ion extraction and transport introduce distortion to the transport of charge state information. It is well known that ion extraction produces a parallel beam of ions only when the perveance condition is met [31], 
i.e. when plasma density, extraction voltage, and geometry of ion optics are chosen appropriately. Overdense extraction has been used to intentionally reduce the noise of ion beams [26]. In figure 10 one can notice that the amplitude of ion spectra is a little greater near the beginning and end of the arc pulse while lower in the center section. This is due to a slightly overdense extraction in the center of the pulse. It is difficult to estimate what percentage of error such perveance mismatch might have caused. Yet, even if near-prefect matching was achieved for average conditions, the issue remains since the plasma density fluctuates, and with density fluctuations the perveance mismatch will fluctuate, too. This represents an accuracy limit for the method but does not change the general findings presented in this and other contributions.

\section{Conclusions}

Summarizing the results of this study, one must first state that fluctuations of ion charge states were always present, even at the shortest time difference between measurements. This finding fits well with previous observations of nanosecond changes in crater development, voltage fluctuations, and light emission and absorption. The fast rate of fluctuations in ion charge state distributions are another indication of fast cathode processes. Yet, the interpretation of fluctuations of ion charge states showed fundamental limitations in using the method for high-resolution plasma diagnostics. These limitations are mainly due to mixing of ion velocities prior to ion extraction. Such mixing causes ion charge state distribution to be correlated on a short time scale, which is especially significant for heavy, slow ions. Additionally, there are some limitations of the method in terms of preservation of ion charge state information due to perveance mismatch at the ion extractor. Still, measurements showed systematic decay of the mean ion charge state after the arc was triggered, and it also showed that the level of charge state fluctuations is material dependent.

\section{Acknowledgements}

This work was supported by the U.S. Department of Energy, Initiatives of Proliferation Prevention, project IPP-LBNL-T2-196, under Contract No. DE-AC03-76SF00098. K.F. acknowledges 
supported by Nippon Sheet Glass Co. Ltd, and G.Y. was supported by a Grant of the President of Russia, Grant number MD-148.2203.02. 


\section{References}

[1] Plyutto A A, Ryzhkov V N, and Kapin A T Sov. Phys. JETP 196520 328-337.

[2] Davis W D and Miller H C J. Appl. Phys. 196940 2212-2221.

[3] Brown I G Rev. Sci. Instrum. 199465 3061-3081.

[4] Anders A Phys. Rev. E 199755 969-981.

[5] Spädtke P, Emig H, Wolf B H, and Oks E Rev. Sci. Instrum. 199465 3113-3118.

[6] Oks E, Yushkov G, Schanin P, and Nikolaev A Rev. Sci. Instrum. 199667 1213-1215.

[7] Bilek M M M, Martin P J, and McKenzie D R J. Appl. Phys. 1998 83 2965-2970.

[8] Rosén J, Anders A, Hultman L, and Schneider J M J. Appl. Phys. 200394 1414-1419.

[9] Paoloni F J and Brown I G Rev. Sci. Instrum. 199566 3855-3858.

[10] Oks E M, Anders A, Brown I G, Dickinson M R, and MacGill R A IEEE Trans. Plasma Sci. 1996 24 1174-1183.

[11] Anders A and Yushkov G Y J. Appl. Phys. 200291 4824-4832.

[12] Brown I G, Galvin J E, MacGill R A, and Wright R T Rev. Sci. Instrum 198758 1589-1592.

[13] Reich H, Spädtke P, and Oks E M Rev. Sci. Instrum. 200071 707-709.

[14] Reece M P Nature 1958181 475-476.

[15] Anders A, Yotsombat B, and Binder R J. Appl. Phys. 200189 7764-7771.

[16] Byon E and Anders A J. Appl. Phys. 200393 1899-1906.

[17] Lunev V M, Padalka V G, and Khoroshikh V M Instrum. Exp. Tech. 197619 1465-1467.

[18] Brown I G, Spädtke P, Rück D M, and Wolf B H Nucl. Instrum. Meth. Phys. Res. A $199029512-$ 20.

[19] Puchkarev V F J. Phys. D: Appl. Phys. 199124 685-692.

[20] Smeets R P P and Schulpen F J H J. Phys. D: Appl. Phys. 198821 301-310.

[21] Anders A IEEE Trans. Plasma Sci. 199927 1060-1067.

[22] Jüttner B J. Phys. D: Appl. Phys. 200134 R103-R123.

[23] Aksenov I I, Konovalov I I, Padalka V G, Sizonenko V L, and Khoroshikh V M Sov. J. Plasma Phys. 198511 787-791.

[24] Tsuruta K, Nakajima M, and Yanagidaira T IEEE Trans. Plasma Sci. 2003 31 953-956. 
[25] Oks E, Yushkov G, Litovko I, Anders A, and Brown I Rev. Sci. Instrum. 2001 73 735-737.

[26] Anders A and Hollinger R Rev. Sci. Instrum. 200273 732-734.

[27] Rosén J, Anders A, Hultman L, and Schneider J M J. Appl. Phys. 200496 4793-4799.

[28] Brown I G and Godechot X IEEE Trans. Plasma Sci. 199119 713-717.

[29] Yushkov G Y, Oks E M, Anders A, and Brown I G J. Appl. Phys. 200087 8345-8350.

[30] Yushkov G Y and Anders A IEEE Trans. Plasma Sci. 199826 220-226.

[31] Forrester A T, Large Ion Beams. New York: Wiley, 1988.

[32] Anders A, Anders S, Jüttner B, and Brown I G IEEE Trans. Plasma Sci. 1993 PS-21 305-311.

[33] Anders A IEEE Trans. Plasma Sci. 200533 accepted for publication.

[34] Jüttner B J. Phys. D: Appl. Phys. 198114 1265-1275.

[35] Puchkarev V F and Murzakayev A M J. Phys. D: Appl. Phys. 199023 26-35.

[36] Anders A, Anders S, Jüttner B, Bötticher W, Lück H, and Schröder G IEEE Trans. Plasma Sci. $199220466-472$.

[37] Molmud P Phys. Fluids 19603 362-366.

[38] Mesyats G A, Explosive Electron Emission. Ekaterinburg: URO Press, 1998. 


\section{Figure Captions}

Figure 1. Simplified schematic of the vacuum arc ion source with a time of flight spectrometer. The new special feature is the option for multiple TOF gate pulses in a very short time period.

Figure 2. Arc current pulse (bottom, $100 \mathrm{~A} / \mathrm{div}$ ) and an example of timing the two probing gate pulses (top, $2 \mathrm{kV} / \mathrm{div}$ ) with respect to the arc pulse and with respect to each other.

Figure 3. Two TOF spectra (lower curve) of a 200 A copper plasma obtained in a single arc pulse by using two gate pulses (upper curve). The charge states are indicated by numbers. The charge state amplitude, which is shown here, is given by the electrical current to the detector, hence each signal needs to be divided by the corresponding charge state number in order to obtain the particle current, which is used to determine plasma composition.

Figure 4. Changes in magnesium ion charge states as a function of time $\Delta t$ elapsed between measurements. Top: charge state $Q=1$, bottom: charge state $Q=2$. For each $\Delta t$, measurements were repeated 25 times, resulting in the scattering of data. Each measurement $(1$, $2, \ldots, 25)$ was assigned a symbol for display and tracking but the reader should focus on the range of data scattering rather than on individual data points. Arc current $150 \mathrm{~A}$; first gate 150 $\mu$ s after arc triggering. The average absolute amplitudes (in arbitrary but same units, at $t \approx 150 \mu s$ ) were about 21 for $Q=1$ and about 61 for $Q=2$.

Figure 5. As figure 4 but for bismuth, with arc current $200 \mathrm{~A}$. The average absolute amplitudes (in arbitrary but same units, at $t \approx 150 \mu s$ ) were about 42 for $Q=1$ and about 28 for $Q=2$.

Figure 6. As figure 4 and 5 but for copper with $200 \mathrm{~A}$ arc current. In contract to $\mathrm{Mg}$ and $\mathrm{Bi}$, copper has 4 ion charge states to be considered. The average absolute amplitudes (in arbitrary but same units, at $t \approx 150 \mu \mathrm{s}$ ) were about $7,68,88,7.5$ for $Q=1,2,3,4$, respectively.

Figure 7. Standard deviation of changes in mean charge state as a function of time difference $\Delta t$ between measuring TOF gate pulses. Each point in this presentation is derived from 25 individual measurements. The lines represent spline fits.

Figure 8. Standard deviation of changes in mean ion charge states for bismuth as a function of time between TOF gate pulses, with the length of the plasma expansion zone (cathode - ion extractor distance) as a parameter. 
Figure 9. Standard deviation of changes of $\mathrm{Mg}$ ion charge states for constant time difference between TOF gate pulses, $\Delta t=220 \mu \mathrm{s}-150 \mu \mathrm{s}=70 \mu \mathrm{s}$, as a function of arc current. The line is calculated assuming 0.8 A per emission site, as explained in section 4 .

Figure 10. Overview of burst of gate pulses (top), time-of-flight ion spectra (center), and arc current pulse (bottom), with low temporal resolution.

Figure 11. As figure 10 but with high time resolution and not showing the complete pulse.

Figure 12 As figure 11, now with sufficiently high time resolution that permitted clear identification and measurement of a charge state distribution belonging to a gate pulse.

Figure 13 Snapshots of single-pulse charge state evolutions, one per element, showing the systematic decay of the mean charge state and the material-dependent level of fluctuation.

Figure 14. Velocity distribution function for magnesium ions in the plasma prior to ion extraction (data from [16]). 